\title{
Orienting of attention with eye and arrow cues and the effect of overtraining
}

\author{
D. Guzzon ${ }^{\text {a,d,* }}$, D. Brignani ${ }^{\text {b }}$, C. Miniussi ${ }^{\text {b,c }}$, C.A. Marzi ${ }^{\text {a }}$ \\ a Department of Neurological and Visual Sciences, Physiology Section, University of Verona, Verona, Italy \\ b Cognitive Neuroscience Section, IRCCS San Giovanni di Dio Fatebenefratelli, Brescia, Italy \\ c Department of Biomedical Sciences and Biotechnologies, National Neuroscience Institute, University of Brescia, Brescia, Italy \\ d Department of General Psychology, University of Padova, Padova Italy
}

\section{A R T I C L E I N F O}

\section{Article history:}

Received 6 October 2009

Received in revised form 22 March 2010

Accepted 25 March 2010

Available online 24 April 2010

\section{PsycINFO classification:}

2346

2343

Keywords:

Eyes

Arrows

Central cues

Orienting of attention

Over-learned association mechanism

\begin{abstract}
A B S T R A C T
In contrast to the classical distinction between a controlled orienting of attention induced by central cues and an automatic capture induced by peripheral cues, recent studies suggest that central cues, such as eyes and arrows, may trigger a reflexive-like attentional shift. Yet, it is not clear if the attention shifts induced by these two cues are similar or if they differ in some important aspect. To answer this question, in Experiment 1 we directly compared eye and arrow cues in a counter-predictive paradigm while in Experiment 2 we compared the above cues with a different symbolic cue. Finally, in Experiment 3 we tested the role of over-learned associations in cueing effects. The results provide evidence that eyes and arrows induce identical behavioural effects. Moreover, they show that over-learned associations between spatially neutral symbols and the cued location play an important role in yielding early attentional effects.
\end{abstract}

(C) 2010 Elsevier B.V. All rights reserved.

\section{Introduction}

A great deal of research has been dedicated to distinguish between different types of orienting of attention. Most of the studies have been carried out with the so-called Posner paradigm (Posner, 1980; Posner, Snyder, \& Davidson, 1980) which is still widely used and in which a central or a peripheral cue precedes the appearance of a peripheral target to which participants are to respond. Targets may appear in the same or in the opposite position with respect to that indicated by the cue and in the former condition they responded more quickly than in the latter presumably because attention is already oriented to the target location. Jonides (1981) and Muller and Rabbitt (1989) tested the effects of different types of attentional cues by comparing the effect of central and peripheral cues under different temporal cuetarget intervals. On the basis of their results they described two major types of attentional control: automatic and voluntary. Traditionally, the former refers to the capture of attention by the sudden appearance of events in the visual field, such as changes in luminance, texture or motion; the latter, instead, refers to a top-down controlled guidance of attention allocation. The effects of automatic orienting have been reported to be short lived (100-300 ms after cue onset), while those

\footnotetext{
* Corresponding author. Dipartimento di Psicologia Generale, Università di Padova, Via Venezia 8, 35131 Padova, Italy. Tel.: +39 0498276920.

E-mail address: daniela.guzzon@unipd.com (D. Guzzon).
}

of voluntary orienting have a longer latency and duration (200$400 \mathrm{~ms}$ ) Muller and Rabbitt (1989). On the whole, the common assumption was that peripheral cues trigger an automatic orienting of attention while central cues induce a controlled orienting mode. In particular, central symbolic cues are typically thought to give rise only to voluntary orienting because they need to be interpreted to extract positional information (Jonides, 1981; Jonides \& Yantis, 1988; Muller \& Findlay, 1988; Muller \& Rabbitt, 1989; Yantis \& Jonides, 1984). This general assumption has recently been challenged by a number of studies showing that biologically relevant cues, such as eyes and head deviation, when centrally presented can automatically trigger orienting of attention (Driver, Davis, Ricciardelli, Kidd, Maxwell, \& BaronCohen, 1999; Friesen \& Kingstone, 1998; Langton \& Bruce, 1999). It is common knowledge that observing another person's gaze leads to a shift of attention in the corresponding direction. To study this phenomenon, modifications of the Posner paradigm have been used in which eyes substitute central arrow cues. The results showed that eye cueing strongly resembles automatic orienting at least for two reasons. First, although centrally presented, eye direction has a rapid cueing effect even when is not predictive of target location (Driver et al., 1999; Friesen \& Kingstone, 1998; Langton \& Bruce, 1999). Second, eye cueing effects are resistant to voluntary control since they occur also when subjects know that targets are more likely to appear in the uncued location (i.e., counter-predictive cue) (Driver et al., 1999; Friesen \& Kingstone, 1998; Friesen, Ristic, \& Kingstone, 2004). 
In comparison with the automatic orienting induced by peripheral cues, eye cueing results in both a prolonged facilitation and a delayed onset of inhibition processes at the gazed-at location. Indeed, orienting to eyes direction persists well beyond a stimulus onset asynchrony (SOA) of $500 \mathrm{~ms}$ (Friesen \& Kingstone, 1998) while reflexive orienting effects usually disappear after 300 ms (Klein, Kingstone, \& Pontefract, 1992). In addition, eyes elicit the so-called inhibition of return (i.e., inhibitory effects for targets appearing at the cued location) only at considerably extended SOAs, such as 2400 ms (Frischen \& Tipper, 2004; Frischen, Smilek, Eastwood, \& Tipper, 2007), whereas typically inhibition of return appears at around $300 \mathrm{~ms}$ with peripheral cues (for a review, see Klein, 2000). These peculiarities led some researchers to suggest that gaze is a special attentional cue because of its biological significance (e.g., Friesen \& Kingstone, 1998, 2003; Langton \& Bruce, 1999). Although other investigators have reported that also arrow cues can induce an automatic orienting of attention even when they are uninformative with regard to target location (Gibson \& Bryant, 2005; Hommel, Pratt, Colzato, \& Godijn, 2001; Ristic, Friesen, \& Kingstone, 2002; Tipples, 2002), studies using a counter-predictive paradigm provided evidence for considering gaze as a special attentional cue (Driver et al., 1999; Friesen et al., 2004). Friesen et al. (2004) showed that only orienting to central arrows can be voluntarily suppressed suggesting that arrowtriggered attention is less automatic than orienting to eyes. They used a modified version of the classic counter-predictive paradigm in which they increased from two to four the number of target locations. Although this procedure was useful to measure voluntary and involuntary orienting it introduced a state of more widely distributed attention thus increasing the difficulty of the task and reducing the strength of the cueing effects. In a recent study, using the same counterpredictive paradigm as that of Friesen et al. (2004), Tipples (2008) found that both eye and arrow cues produce qualitatively similar types of orienting. These data seem to provide concluding evidence that gaze is not different from other types of cues although some methodological problems remain. Neither Tipples (2008) nor any of the previously mentioned studies have directly compared the attentional effects of gaze and arrow cues. Tipples (2008) and Friesen et al. (2004) tested gaze and arrow effects in isolation, that is, in separate experiments with different groups of subjects. This procedure does not allow a direct statistical comparison between the attentional effects induced by the two cues which is a crucial step to conclude for a similarity or difference between them. In the present study we compared the cueing effects induced by eyes and arrows within the same group of subjects and the same experiment, with the specific aim of assessing whether they induce or not the same attentional orienting.

In the first experiment we investigated whether eye and arrow cues trigger a similar attentional shift in a counter-predictive paradigm. In particular, with this paradigm we tested the "intentionality criterion" proposed by Jonides (1981) which postulates that an automatic process must not be amenable to voluntary control. Previous studies using this kind of paradigm (e.g. Driver et al., 1999; Friesen, Ristic, \& Kingstone, 2004; Tipples, 2008) yielded inconsistent results that left the question unsolved. In the light of these uncertainties, we were interested to verify whether the coexistence of automatic and voluntary orienting gives rise to similar advantages in cued but non predicted and in non cued but predicted locations, depending on the cue-target interval.

In the second experiment we tested the time course of the attentional orienting triggered by eyes and arrows in a predictive paradigm. This task required a voluntary top-down control of attention which at short SOAs could be affected by automatic effects or by an overlearned association mechanism. In order to have a real volitional baseline condition we compared the effect of eyes and arrows with those of a novel symbolic cue. Some researchers have recently proposed that all conventional communicative signals, including eyes and arrows, induce an automatic-like orienting of attention as a consequence of an over-learned association between their symbolic meaning and the locations to which they refer (Hommel et al., 2001; Vecera \& Rizzo, 2004,
2006). Indeed, it happens daily that the presentation of these signals is followed by consequences at the indicated location and this creates a strong association inducing an instantaneous orienting of attention toward the relevant location. Consistently with this hypothesis, in a previous study (Brignani, Guzzon, Marzi \& Miniussi, 2009) we provided evidence that orienting triggered by eyes and arrows engages the same cortical network which includes the orbitofrontal cortex, an area clearly involved in association learning.

In order to test the hypothesis that over-learned association mechanisms play a key role in the automatic-like orienting of attention induced by eye and arrow cues (Hommel et al., 2001; Vecera \& Rizzo, 2004, 2006), we performed a third experiment in which subjects were trained with a novel symbolic cue. If practise is crucial in establishing early cueing effects, we would expect a post-training improvement in performance in the cueing task.

\section{Experiment 1}

Orienting of attention is considered automatic if cannot be suppressed at short SOAs and if occurs regardless of participants' volition. Peripheral cues, for instance, can attract spatial attention even when participants are told that targets are more likely to appear on the opposite side to the peripheral cues. This counter-predictive paradigm (Posner, Cohen, \& Rafal, 1982) is based on the following assumption: attention is first involuntary attracted to the location of the peripheral event and then, as SOA increases, participants are gradually able to voluntary shift their attention to the side where the target is expected to appear. Therefore, at short SOAs faster reaction times (RTs) are observed for the location where peripheral cues occur, while at longer SOAs RTs become faster for the contralateral location.

Only three previous studies used the counter-predictive paradigm to investigate the attentional orienting induced by eyes and arrows (Driver et al., 1999; Friesen et al., 2004; Tipples, 2008). In Driver et al., 1999 (Experiment 3) only gaze orienting was studied and the results indicated that at $300 \mathrm{~ms}$ of cue-target interval subjects were faster to detect targets appearing at cued locations even if targets were unlikely to appear there. Friesen et al. (2004) used a modification of the counter-predictive orienting task with both gaze and arrow cues in two different experiments. Targets could appear in either a cued location (gaze-at or pointing at location i.e., $8 \%$ of trials) or in a predicted location (opposite to the gaze-at or pointing at location i.e., $75 \%$ of the trials) or in two locations that were neither predicted nor cued ( $16 \%$ of the trials). These latter locations were considered as the new baseline to obtain an evaluation of voluntary and reflexive orienting: the former through the comparison between predicted and baseline locations, the latter between cued and baseline locations. The authors found an involuntary effect with gaze cue starting at $105 \mathrm{~ms}$ of SOA while failed to observe reflexive effects with arrow cues at any SOAs. Therefore, they concluded that the arrow-cueing effect was more vulnerable to top-down control than the gaze-cueing effect.

These findings have long been used to support the idea that eyes, but not arrows, trigger an automatic-like shift of attention. However, Tipples (2008) has recently found that also arrow cues induce involuntary effect at $105 \mathrm{~ms}$ of SOA by using the same counter-predictive paradigm of Friesen et al. (2004). These contrasting results have increased our uncertainty as to whether gaze and arrows yield similar cueing effects. In addition, it is important to note that in all these previous studies the cueing effects induced by eyes and arrows have never been directly compared within the same group of subjects and the same experiment.

The aim of Experiment 1 was to investigate whether the attentional orienting induced by eyes and arrows is equally affected by top-down control. In order to highlight possible differences between the two cues, the same group of subjects performed the counter-predictive cueing paradigm with both eyes and arrows as central cues. We used the classic counter-predictive paradigm (Posner et al., 1982) with only two possible positions where targets could appear: a cued location (gaze- 
at or pointing at location) and a predicted location (opposite to the gazeat or pointing at location). Participants were informed that the target was four times more likely to appear at the location opposite to where the eyes were looking, or the arrows were pointing, and that the best strategy was to voluntarily contrast the cued position. We checked the time course of the attentional orienting at the two typically shortest (50 and $100 \mathrm{~ms}$ ) and intermediate (300 and $500 \mathrm{~ms}$ ) SOAs.

\section{Method}

\subsection{Participants}

Twelve students, ( 6 females) ranging in age from 20 to 30 years ( mean $=23.92 ; \mathrm{SD}=3.20$ ), and naïve to the aims of the study, participated in this experiment. All were right handed $(+98 \%)$ as tested with the Edinburgh Handedness Inventory Test (Oldfield, 1971) and reported normal or corrected-to-normal visual acuity. They were paid and gave informed consent (Oldfield, 1971).

\subsection{Apparatus and stimuli}

The experiment was controlled by the E-Prime software (Version 1.1 for Windows) using for stimulus presentation a monitor with a resolution of $800 \times 600$ pixels, colour depth at 24 bit, and a refresh rate of $85.057 \mathrm{~Hz}$. The time of presentation of each stimulus was adapted to the refresh rate of the monitor and is rounded here for simplicity. Stimuli were created with a software for image processing and then saved in the grey scale. The two types of central cues included two schematic eyes and two arrows both directed either to the right or to the left (see Fig. 1a and b). Eyes and arrows had the same size $\left(3.39^{\circ} \times 0.90^{\circ}\right)$ and were presented on a black background with a grey fixation dot in the middle. The pupils of the schematic eyes were black filled-in circles pointing to the left or right. The target stimulus was a black and white checkerboard that subtended a visual angle of $2.82^{\circ}$ and was presented inside one of two peripheral boxes $\left(2.9^{\circ}\right)$ along the horizontal meridian centred at $7.5^{\circ}$ from the fixation point.

\subsection{Design and procedure}

Each participant sat in a dimly illuminated room with the head kept at a distance of $57 \mathrm{~cm}$ from the computer monitor by means of a head rest. Participants performed two experimental sessions, one for each type of cue (eyes, arrows). The order of the sessions was counterbalanced across participants. Participants were required to fixate a dot presented at the centre of the display and to detect the appearance of the target by pressing the spacebar of the computer keyboard as quickly as possible. Each trial began with a fixation point (1500 ms) followed by a central cue (eyes, arrows). The cue itself was on until offset of the target (exposure duration: $50 \mathrm{~ms}$ ), which was presented at various randomized cue-target intervals $(50,100,300$ or $500 \mathrm{~ms}$ ). The target was followed by a blank screen until the response was performed or for $1000 \mathrm{~ms}$. All the targets appeared inside one of

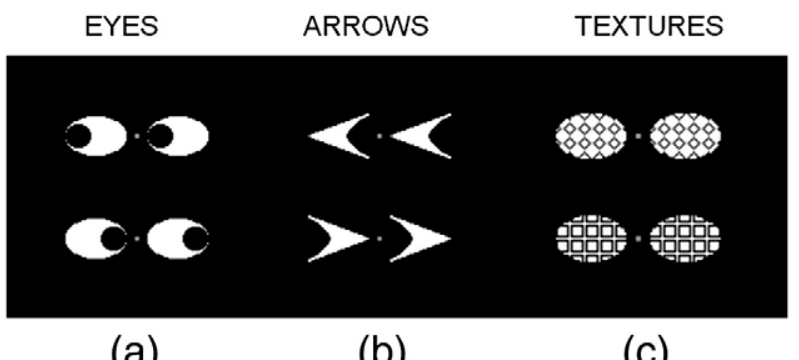

(a)

(b)

(c)

Fig. 1. Rendering of the schematic stimuli used as central cues. Leftward and rightward eyes, arrow and texture cues are presented from the left respectively.

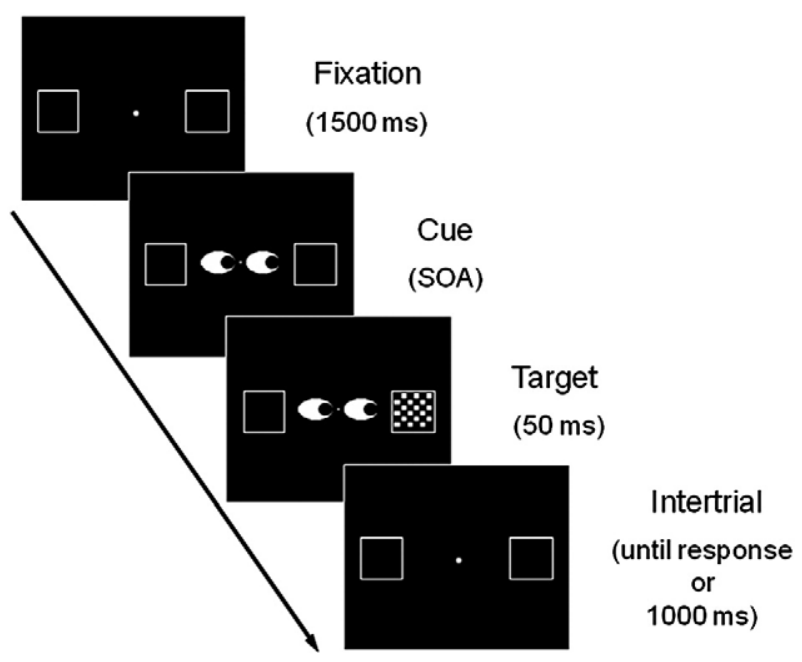

Fig. 2. Illustration of the trial sequence. Each trial began with the presentation of a fixation point with two lateral boxes for $1500 \mathrm{~ms}$. Then a central cue (eyes, arrows or textures) was presented for a variable time, that corresponded to an SOA of 50, 100, 300, and $500 \mathrm{~ms}$ in Experiment 1 and of 50, 100, 150, and $200 \mathrm{~ms}$ in Experiment 2. To follow, a target appeared to the left or to the right of the central cue for $50 \mathrm{~ms}$.

two peripheral boxes which were present during the whole trial. The sequence of events for each trial is shown in Fig. 2.

All cues were counter-predictive, that is, in $80 \%$ of the trials they signalled that the target location was opposite to where eyes were looking, or arrows were pointing (predictive trials). In the remaining $20 \%$ of the trials, targets appeared congruently to the location indicated by the cues (unpredictive trials). Before the start of each session, participants were told that $80 \%$ of the time cues indicated the opposite side to the target appearance, so, in order to perform the task effectively, they had to shift their attention to the other side. The four SOAs $(50,100$, 300 , and $500 \mathrm{~ms}$ ) were presented randomly and with equal probability in each session.

Each of the two experimental sessions consisted of 12 practice trials followed by 8 blocks, each one composed by 80 trials: 64 predictive trials and 16 non-predictive trials. Moreover, 4 catch trials were presented in each block. After each block there was a brief resting interval. The whole testing session lasted about one hour, including instructions and short breaks. During each session the responding hand was changed after four blocks of trials according to a counterbalanced order: one half of the participants began with the right hand in the first four blocks and with the left hand in the last four blocks, and vice versa for the other half.

Anticipations (i.e., pressing the spacebar before the appearance of the target, or on catch trials) and omissions (no response) were classified as errors and were excluded from the analyses. Too short or too long RTs (RT $>$ or $<$ than \pm 2 standard deviation cut-off procedure) were excluded as well. Each type of error accounted for less than $1.41 \%$ of the total number of trials. Since these rates were low, errors were not further analyzed.

An ANOVA was carried out on mean RTs, with Cue (eyes and arrows), SOA (50, 100, 300 or $500 \mathrm{~ms}$ ) and Trial Type (predictive and unpredictive trials) as within-subject variables. Selected two-sample comparisons were performed by means of $t$ tests for paired data with Bonferroni correction when appropriate.

\section{Results}

The ANOVA showed a main effect of Trial Type, $F(1,11)=25.4$, MS $=11167, p=0.0004$, indicating a significant difference in RTs between predictive $(277 \mathrm{~ms}$ ) and unpredictive $(292 \mathrm{~ms})$ trials. According to the instructions, participants oriented their attention towards the location where the target was more likely to appear (i.e., the side opposite to the eyes and arrow -pointed locations). In particular, the significant SOA $\times$ Trial Type interaction, $F(3,33)=27.09$, MS $=2691$, 
$p=0.0001$, showed that participants were able to orient their attention towards the predicted location starting from $100 \mathrm{~ms}$ of SOA (see Fig. 3 and Table 1). This is shown by the fact that the difference in RTs between predictive and non-predictive trials was significant at $100 \mathrm{~ms}$ $(p=0.03), 300 \mathrm{~ms}(p=0.0004)$ and $500 \mathrm{~ms}(p=0.0001)$, but not at $50 \mathrm{~ms}$ of SOA $(p=0.8)$. A significant main effect of SOA, $F(3,33)=30.35$, $\mathrm{MS}=18561, p=0.0001$, also emerged reflecting a cue-target foreperiod effect with RTs becoming shorter as SOA increased. Finally, the Cue $\times$ SOA interaction was significant, $F(3,33)=7.79, \mathrm{MS}=404$, $p=0.0005$, indicating similar responses for the two cues at all but $50 \mathrm{~ms}$ of SOA. At this shortest SOA, reaction times were faster with arrow (304 ms) than with gaze cues $(312 \mathrm{~ms})(p=0.006)$, independently from Trial Type (predicted or non predicted). Indeed, there was no significant interaction between Cue, Trial Type and SOA $(p=0.3)$. Thus, somewhat surprisingly, participants showed the same early ability to orient their attention according to the incongruent and predictive cues both with eyes and arrows.

To verify whether this result was related to a practice effect we tested the possible difference between the first four and the last four blocks of each session. An ANOVA was conducted with five factors: Block (first four and last four), Cue (eyes, arrows), SOA (50, 100, 300 or $500 \mathrm{~ms}$ ), and Trial Type (predicted and not predicted), as withinsubject variables. The ANOVA confirmed the results described above and showed no interactions related to a practice effect (Block $\times$ Trial Type, $p=0.09$; Block $\times$ Trial Type $\times$ SOAs, $p=0.27$ ) but only a main effect of Block, $F(1,11)=7.63$, MS $=4963, p=0.02$, with the first four blocks $(282 \mathrm{~ms}$ ) yielding faster RTs than the latter four blocks (288 ms).

\section{Discussion}

In this experiment a counter-predictive paradigm was used to investigate whether eyes and arrows trigger a similar automatic-like attentional shift. Automatic orienting of attention is classically considered to resist volitional control, at least at short SOAs (Jonides, 1981) and therefore, in the present experiment targets were expected to be detected more quickly at short SOAs when they appeared at eyes-at and arrow-pointing locations and at long SOAs when they appeared at the opposite side.

Surprisingly, the results of this experiment highlighted an early top-down control of attention (i.e., from $100 \mathrm{~ms}$ ) with both central cues. This effect was expressed by an early advantage for the predicted, although spatially incongruent, positions that began at $100 \mathrm{~ms}$ of SOA and persisted at all longer delays $(300,500 \mathrm{~ms})$. An

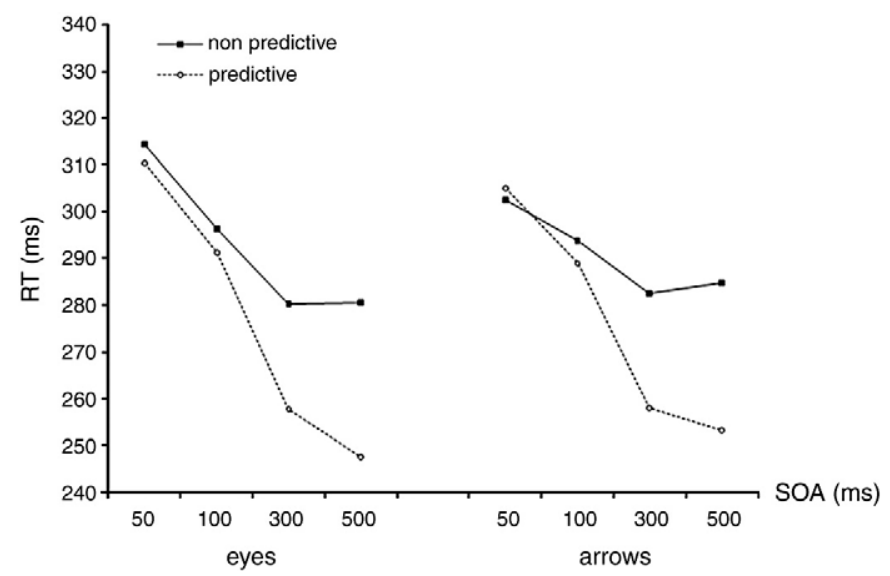

Fig. 3. Mean reaction times (RT) for the counter-informative cues (eyes and arrows) as a function of cue-target stimulus onset asynchrony (SOA) and trial type (predicted and not predicted) in Experiment 1. In predicted and not predicted trials targets occurred respectively at the location opposite and congruent to where eyes were looking or arrows were pointing.
Table 1

Mean reaction times (ms) and standard deviations (SD) for each cue, trial type and SOA in Experiment 1.

\begin{tabular}{|c|c|c|c|c|c|}
\hline \multicolumn{6}{|l|}{ SOA } \\
\hline \multirow[b]{2}{*}{ Cue type } & \multirow[b]{2}{*}{ Trial type } & \multirow{2}{*}{$\frac{50}{\text { Mean (SD) }}$} & \multirow{2}{*}{$\frac{100}{\text { Mean (SD) }}$} & \multirow{2}{*}{$\frac{300}{\text { Mean (SD) }}$} & \multirow{2}{*}{$\frac{500}{\text { Mean (SD) }}$} \\
\hline & & & & & \\
\hline \multirow[t]{2}{*}{ Eyes } & Non-predictive & $314,4(8.5)$ & $296.2(7.2)$ & $280.3(8.9)$ & $280.5(7.3)$ \\
\hline & Predictive & 310.4 (6.6) & $291.4(6.9)$ & $257.8(6.9)$ & $247.8(7)$ \\
\hline \multirow[t]{2}{*}{ Arrows } & Non-predictive & 302.6 (7.9) & $293.75(9.5)$ & $282.6(8.7)$ & 284.9 (8.9) \\
\hline & Predictive & $305(7.6)$ & $289.1(7.7)$ & $258.3(7.8)$ & $253.3(7.4)$ \\
\hline
\end{tabular}

advantage for the non predicted, but cued trials was never observed, neither at $50 \mathrm{~ms}$.

These results may appear in contrast with previous studies showing that over-learned stimuli, such as arrows, eyes, direction words and manual gestures, induce an automatic-like shift of attention. Indeed, in these studies participants responded more quickly when targets appeared at cued locations even though the cues carried no predictive information (Driver et al., 1999; Friesen \& Kingstone, 1998; Hommel et al., 2001; Langton \& Bruce, 1999). However, it is important to note that so early voluntary effects have been already reported in literature even with peripheral cues (Folk, Remington, \& Johnston, 1992; Warner, Juola, \& Koshino, 1990; Yantis \& Jonides, 1990; Warner et al., 1990), suggesting that the control of attention may be more early and profoundly influenced by volitional factors than it was classically thought (Jonides, 1981). Moreover, Tipples (2008) also reported early voluntary effects in a counterpredictive paradigm, starting from $105 \mathrm{~ms}$. In the present experiment no effect was observed at $50 \mathrm{~ms}$ of SOA and it may be hypothesized that both voluntary and automatic orienting occurred concurrently at this short SOA cancelling each other out. However, the important result for the purpose of the present experiment, was that the same effects were found with both eye and arrow cues.

The time course of voluntary orienting to counter-predictive eyes and arrows is different here in comparison with previous studies of Driver et al. (1999) and Friesen et al. (2004) in which participants were able to move their attention to the predicted location only starting from $600 \mathrm{~ms}$ after cue presentation. This discrepancy might partly be accounted for by the kind of cue stimuli employed. In Driver et al.'s (1999) experiment a realistic face pointing straight ahead was used, with eyes gazing to the left or right. The coding of this stimulus presumably took more time than for the simple schematic eyes used in our experiment. Indeed, it has been shown that uninformative schematic faces produce stronger orienting effects than real photographs of faces even at $14 \mathrm{~ms}$ of SOA (Hietanen \& Leppanen, 2003), and that facial features, like head contour and nose angle, affect eye gaze perception (Langton, Honeyman, \& Tessler, 2004). However, a comparison between complex and schematic stimuli has never been carried out in a counter-informative paradigm, so no firm conclusion can be drawn. In Friesen et al.'s (2004) study schematic eyes and arrows were used but their different saliency could explain the reason why only the former induced an automatic orienting. Indeed, in the eyes condition two eyes appeared on a round face while in the arrow condition two ticks appeared at the end of a cross centred within a circle; the former were surely more impressive than the latter. On the contrary, Tipples (2008), who used the same paradigm but a slightly more effective arrow configuration, obtained similar effects with both eyes and arrow cues.

We believe that our procedure, assuring a perceptual homogeneity between eye and arrow cues and a direct comparison between them, provides strong evidence that highly stylized eyes and arrows produce similar effects of attentional orienting in a counter-predictive paradigm. We believe that eyes and arrows are able to induce similar attentional effects also in the "real world", when they show the same degree of saliency. For instance, a flashing arrow indicating the correct 
way during road works may induce a similarly strong attentional shift as the averted eyes of a person standing in front of us signalling to watch a person next to us without being noticed.

\section{Experiment 2}

The findings of Experiment 1 suggest that, despite their greater biological relevance, eyes induce the same attentional effects as arrow cues. In addition, they support the claim that voluntary control of attention acts very early with either eye or arrow cues as participants were able to orient attention opposite to the direction indicated by the cues starting from $100 \mathrm{~ms}$ after cue presentation. This is contrary to the assumption that arrows and eyes induce an automatic-like shift of attention. Indeed a facilitation was expected at short SOAs for the cued locations, even if non predicted, if these cues triggered a strong automatic shift of attention. We found a very early endogenous control of attention that has already been observed in the literature (Folk, Remington, \& Johnston, 1992; Warner, Juola, \& Koshino, 1990; Yantis \& Jonides, 1990; Warner et al., 1990). In a previous study, we compared the cueing effects of eyes and arrows with those of a novel symbolic cue devoid of any intrinsic directional meaning, i.e. a texture cue (Brignani et al., 2009). We performed two experiments with the same predictive cues but different SOAs, namely: 200, 700 and $900 \mathrm{~ms}$ in Experiment 1, and 100, 200 and $700 \mathrm{~ms}$ in Experiment 2. In the first experiment, textures induced cueing effects starting from an SOA of $200 \mathrm{~ms}$, consistently with an early involvement of the voluntary control of attention. However, in the second experiment, at variance with eyes and arrows, no significant effects were found with the texture cue at the shortest SOA of $100 \mathrm{~ms}$ and only a trend was observed at $200 \mathrm{~ms}$, suggesting a difference between the attentional orienting induced by eyes and arrows in comparison to the endogenous cue. A systematic exploration of the cueing effects induced by the textures as a function of SOAs was not performed, since that study was aimed at investigating the electrophysiological correlates of attentional orienting by arrows and eye gaze.

Thus, the aim of the present experiment was to compare the time course of the attentional orienting induced by eyes, arrows and the texture cue by using short SOAs (50,100, 150, $200 \mathrm{~ms})$. We wanted to find out whether over-learned cue stimuli actually trigger a faster cueing effect with respect to a novel cue or whether, in contrast, volitional control can affect attentional shift at so early SOAs with every cue type. Participants were informed about the directional meaning of the texture cues at the beginning of the experiment but they did not undergo any training procedure. The novel cue clearly needed to be informative and all the cues were predictive of the target location in $80 \%$ of the trials. We predicted that eye and arrow cues would yield a larger cueing effect in comparison to textures if, in addition to volitional control, other mechanisms (e.g., perceptually or over-learned characteristics) affected the orienting induced by these cues.

\section{Method}

\subsection{Participants}

Twelve students ( 6 females), in the age range of 20-35 years (mean $=24.67 ; \mathrm{SD}=4.77$ ) and naïve to the aims of the study, took part in the experiment as paid volunteers. All were right handed $(+98 \%)$ as tested with the Edinburgh Handedness Inventory Test (Oldfield, 1971), had normal or corrected-to-normal visual acuity and gave informed consent.

\subsection{Apparatus and stimuli}

The apparatus was identical to that used in Experiment 1 except that we added a third non-directional cue (texture) of the same size as those of the two previous cues (eyes and arrows) (see Fig. 1c).

\subsection{Design and procedure}

Experimental conditions, room illumination and the distance from the computer monitor, as well as, the sequence of events in each trial were the same as in Experiment 1. But in this case we used four short SOAs (50, 100, 150 or $200 \mathrm{~ms}$ ), an additional non-directional cue (textures) and a predictive paradigm. Participants were informed that in most of the trials targets would appear where indicated by the direction of eyes and arrows or congruently with the texture assigned meaning.

The three central cues were presented in three different sessions each and included two schematic eyes, two arrows, both directed either to the right or to the left, or two different textures (rhombus and squares) within two ellipses, to which a directional meaning was assigned (see Fig. 1). The association rhombus-right and square-left was assigned to half of the participants while the reverse association was assigned to the other half. The sequence of presentation of the three cue types was counterbalanced across participants. All cues were informative: in $80 \%$ of the trials they indicated the correct target location (valid trials), while in the remaining $20 \%$ of the trials they indicated the incorrect location (invalid trials).

Each of the three experimental sessions consisted of 12 practice trials, followed by 8 blocks of 80 trials each including 64 valid and 16 invalid trials. Furthermore, 8 catch trials in which no target appeared were presented in each block.

Anticipations and false alarms (i.e., pressing the spacebar before the appearance of the target, or on catch trials) and omissions (no response) were classified as errors, and excluded from the analyses. Too short or too long RTs (RT $>$ or $<$ at \pm 2 standard deviation cut-off procedure) were excluded from further analysis. Each type of error accounted for less than $3.74 \%$ of the total number of trials. Because these rates were low, errors were not further analyzed.

An ANOVA was carried out on mean RTs, with Cue (eyes, arrows and textures), SOA (50, 100, 150 or $200 \mathrm{~ms}$ ) and Trial Type (valid and invalid trials) as within-subject factors. Selected two-sample comparisons were performed by means of $t$ tests for paired data, with Bonferroni correction as appropriate, in case of multiple comparisons.

\section{Results}

The main effect of Trial Type was significant, $F(1,11)=61.07$, $\mathrm{MS}=16650, p=0.0001$, with a speed advantage in RT for valid (299 ms) versus invalid trials $(314 \mathrm{~ms})$. The significant Cue $\times$ Trial Type interaction, $F(2,22)=29.1$, MS $=2886, p=0.0001$, revealed that the Trial Type effect was significant with eyes and arrow cues $(p=0.0001)$ but not with texture cues $(p=1.00)$.) In addition, valid trials were slower with textures than with eyes $(p=0.04)$ and arrows $(p=0.008)$, whereas there were no difference between invalid trials. Interestingly, the significant interaction Cue $\times \mathrm{SOA} \times$ Trial Type, $F(6,66)=3.81$, MS $=262, p=0.002$, revealed that the cueing effect was significant with eye and arrow cues at all SOAs, while it never reached a significant value with the texture cues (see Fig. 4 and Table 2). Moreover the planned t tests confirmed the difference in RT between the different cues for the valid trials only at each SOA. The ANOVA showed also a significant main effect of SOA, $F(3,33)=21.99$, MS $=16719, p=0.0001$, reflecting a typical cue-target foreperiod effect with RT becoming shorter as SOA increased.

\section{Discussion}

In this experiment, the attentional orienting induced by eyes and arrows was studied in comparison with that induced by a novel symbolic cue. Our prediction was that the two former cues should have induced a faster attentional orienting with respect to the latter. This prediction was confirmed since a validity effect appeared early (50 ms) with the two over-learned cues while was not present with the texture cue at any SOAs. 


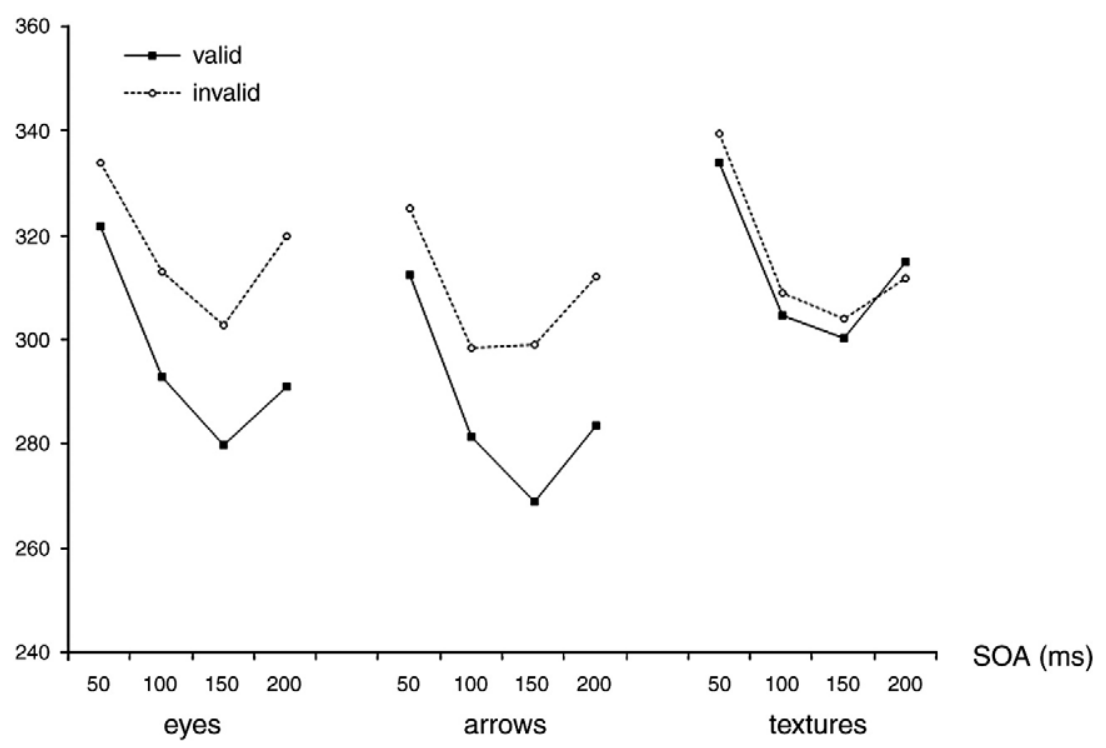

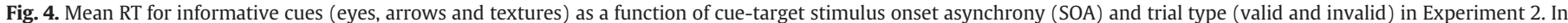
valid and invalid trials targets occurred respectively at the location where eyes were looking or arrows were pointing and opposite to them.

Importantly, also in this experiment, we found no significant differences between eye and arrow cues, neither in the time course nor in the overall magnitude of the validity effect. These results suggest that participants were able to orient their attention according to the direction indicated by both eyes and arrows already at $50 \mathrm{~ms}$ of SOA, that is, earlier compared with the previous experiment, in which a facilitation effect was observed in predicted trials starting from $100 \mathrm{~ms}$. These faster cueing effects can be ascribed to the predictive paradigm used in which voluntary and automatic orienting are likely to occur concurrently thus increasing their efficacy. In particular, the automatic-like attentional orienting induced by eyes and arrows was strengthened by the internal goal to orient to the side indicated by the cue as suggested by the instructions. In experiment 1 , on the contrary, at $50 \mathrm{~ms}$ of SOA voluntary and automatic orienting probably cancelled each other, producing no effect. To explain the automatic-like orienting effects yielded by eyes and arrows, as well as by other stimuli such as direction words and manual gestures, an association learning mechanism has been proposed (Vecera \& Rizzo, 2006). In daily life, these associations involve a relationship between cues and signalled locations. When a central symbolic cue is used in an experimental setting it is commonly assumed that participants process the central cue as a symbol, form a plan to allocate attention according to the information carried by the symbol and then execute that plan. All these steps are supposed to occur very quickly with over-learned stimuli, producing the automatic-like attentional effects.

In the present experiment the novel non-directional cue never produced a cueing effect. Most likely, the voluntary orienting required by the predictive paradigm was not enough to produce an attention

Table 2

Mean reaction times (ms) and standard deviations (SD) for each cue, trial type and SOA in Experiment 2.

\begin{tabular}{|c|c|c|c|c|c|}
\hline \multicolumn{6}{|l|}{ SOA } \\
\hline \multirow[b]{2}{*}{ Cue type } & \multirow[b]{2}{*}{ Trial type } & \multirow{2}{*}{$\frac{50}{\text { Mean (SD) }}$} & \multirow{2}{*}{$\frac{100}{\text { Mean (SD) }}$} & \multirow{2}{*}{$\begin{array}{l}150 \\
\text { Mean (SD) }\end{array}$} & \multirow{2}{*}{$\frac{200}{\text { Mean (SD) }}$} \\
\hline & & & & & \\
\hline \multirow[t]{2}{*}{ Eyes } & Valid & $321.7(15.6)$ & $292.9(14.1)$ & 279.7 (13.6) & $291(12.2)$ \\
\hline & Invalid & $333.9(18.1)$ & $312.9(16.2)$ & 302.7 (16.4) & 319.8 (14.7) \\
\hline \multirow[t]{2}{*}{ Arrows } & Valid & $312.5(16.9)$ & $281.2(15.6)$ & $268.8(14.5)$ & $283.5(12)$ \\
\hline & Invalid & $325.1(21.7)$ & $289.4(16.8)$ & 298.9 (15.7) & $312(14.7)$ \\
\hline \multirow[t]{2}{*}{ Textures } & Valid & $333.9(20.1)$ & 304.7 (19.1) & $300.2(17.7)$ & $314.98(16.1)$ \\
\hline & Invalid & 339.4 (20.9) & $309.1(20.8)$ & $303.8(16.3)$ & $311.6(14.7)$ \\
\hline
\end{tabular}

shift at short SOAs with this kind of cue without any pre-learned cuedirection association. Evidence provided by our previous work (Brignani et al., 2009) suggests that the SOA of $200 \mathrm{~ms}$ is borderline for textures to trigger an attentional shift, depending on the sequence of SOAs employed in the experiment, i.e. the reaction time benefits found reflect a rigid process, with a fixed optimal time course (Teichner, 1954). It is reasonable to suppose that the sequence of short SOAs used in the present experiment may have discouraged the subjects to learn the texture-direction association.

On the whole, the findings of experiment 2 support the view that both eyes and arrows, as over-learned cue stimuli, evoke a peculiar type of attentional orienting, which is not induced by novel cue stimuli.

\section{Experiment 3}

Experiment 2 showed that eyes and arrows induce a very early orienting of attention while a novel symbolic cue (texture) is not able to generate cueing effects at the same short cue-target intervals in a predictive paradigm. However, the results of experiment 1 suggest that arrows and eyes do not induce an automatic shift of attention. As already mentioned, an association learning mechanism has been proposed (Vecera \& Rizzo, 2006) to explain the early automatic-like orienting effects induced by these cues. Eyes and arrows, indeed, can be considered special cue stimuli since a strong effect of learning during all life may have influenced and consolidated the cue-direction association. To date, however, this has never been demonstrated. Considering the entire process required to perform a cued task, the final outcome obtained with over-learned cue stimuli may benefit from a facilitation acting at two different stages: at decoding of the cue, allowing a fast extraction of the directional meaning, or at execution of the attentional shift. In both cases, over-learned cues become rapidly available for deploying attention.

The aim of Experiment 3 was to evaluate the role of learning in the establishment of the attentional effects induced by central cues, by training participants to consolidate new cue-direction associations. If practice is a crucial factor in orienting with central cues a better performance should follow extensive practice. In principle, however, we expect an improvement in the time course of the cueing effects also after a relatively short period of learning. We tested the performance with the novel texture cue in a predictive cueing 
paradigm similar to that used in Experiment 2 before and after three weeks of practice.

\section{Method}

\subsection{Participants}

Six subjects, including three authors, (3 females) ranging in age from 25 to 47 years (mean $=33.5 ; \mathrm{SD}=8.38$ ) participated in this experiment. All participants were right handed according to the Edinburgh Handedness Inventory Test (Oldfield, 1971) (+90.5\%), with normal or corrected-to-normal visual acuity and gave informed consent.

\subsection{Apparatus and stimuli}

The apparatus was identical to that used in Experiments 1 and 2, except that we used a different computer screen (17-in. Sonic P70) with a same resolution of $800 \times 600$ pixels, colour depth at 16 bit, and a refresh rate of $60.344 \mathrm{~Hz}$ for the stimulus presentation. In the preand post-test sessions texture cues and target stimuli were identical to those used in Experiment 2. Also in the training sessions texture cues were the same as in Experiment 2, while target stimuli were two letters $(\mathrm{C}$ or $\mathrm{O})$ with the same size $\left(0.4^{\circ} \times 0.4^{\circ}\right)$. They were presented on a black background inside one of two peripheral boxes $\left(2.9^{\circ}\right)$ along the horizontal meridian centred at $7.5^{\circ}$ from the fixation point.

\subsection{Design and procedure}

Experimental conditions, room illumination and the distance from the computer monitor were the same as in Experiments 1 and 2 . Participants performed a pre-training session, a daily training for 3 weeks (15 days) and finally a post-training session. Pre- and posttraining sessions consisted of a predictive paradigm identical to that used in Experiment 2. Participants were required to fixate a dot in the centre of the display and to detect the appearance of a checkerboard presented inside one of two peripheral boxes. They were informed that in most of the trials (80\%) targets would appear congruently with the texture-direction association (valid trials), while in the remaining $20 \%$ of the trials they would appear in the opposite location (invalid trials). The same short SOAs as in Experiment 2 were used $(50,100$, 150 or $200 \mathrm{~ms}$ ).

In the training sessions participants were required to fixate a central dot and to discriminate between two capital letters ( $\mathrm{C}$ or $\mathrm{O}$ ) appearing in the periphery. We used a discrimination task in the training session since it required a higher attentional involvement in orienting attention. Indeed this assured that subjects oriented attention to the cued position in order to execute correctly the task. Moreover, a choice task enabled to introduce a feedback on the accuracy of response. The sequence of events for each trial is shown in Fig. 5 . Each trial began with a fixation point $(1500 \mathrm{~ms})$ followed by a central texture cue which was on until the offset of the peripheral target (50 ms exposure duration). The target was followed by a blank screen until response or for $1000 \mathrm{~ms}$. A feedback about speed of RT and accuracy of the response was provided at the end of each trial for $2000 \mathrm{~ms}$.

Participants were asked to respond as fast and as accurately as they could by pressing with two different fingers of the right hand one of the two keys corresponding to the target letters, independently of the side where the target appeared. Every participant was assigned a texture-direction association (i.e., rhombus-right and square-left or the reverse) and this association was kept constant in all experimental sessions (pre- post-training and training). All cues were predictive in $100 \%$ of the trials, that is, they correctly indicated the location where targets would appear. This was done in order to establish a conditioning between a central symbol and the appearance of an

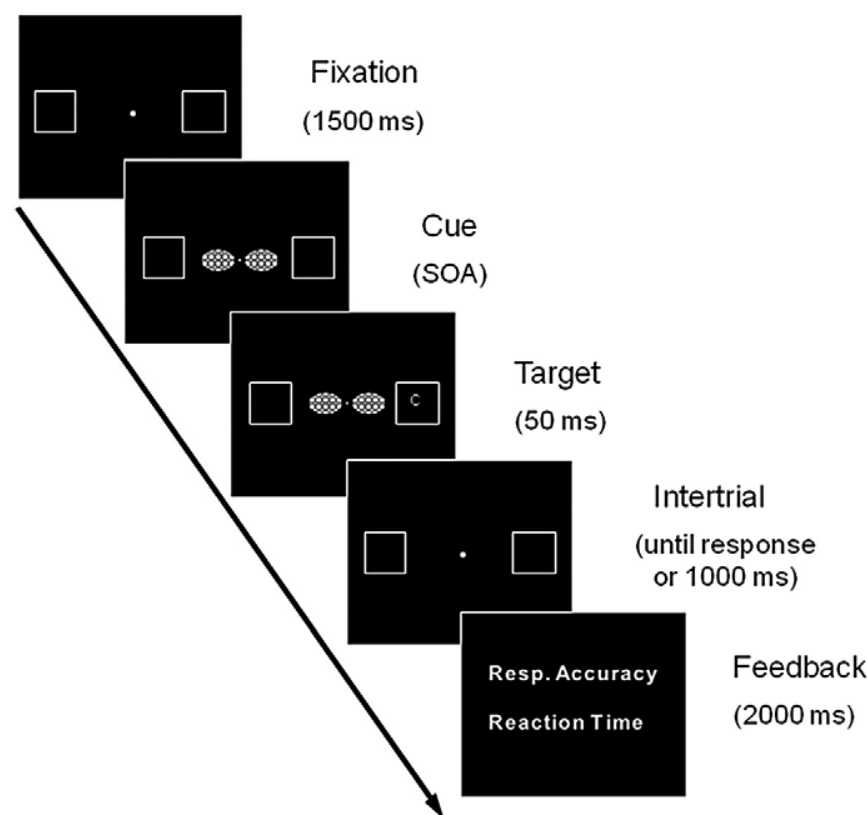

Fig. 5. Illustration of the trial sequence of the Training procedure. Each trial began with the presentation of a fixation point with two lateral boxes for $1500 \mathrm{~ms}$. Then a central texture cue was presented for a variable time, corresponding to an SOA of 100, 200, 300, and $400 \mathrm{~ms}$. To follow, a target letter ( $\mathrm{C}$ or $\mathrm{O}$ ) appeared at the instructed location for $50 \mathrm{~ms}$. In the example here reported the association rhombus-left and square-right was assigned. Participants were required to perform a discrimination between the two letters. The target was followed by a blank screen and by a feedback about speed and accuracy of the response.

event in periphery as is believed to occur with eyes and arrows. In addition, target letters had a size and a duration which required an effective attentional shift in order to be detected. Before the training sessions participants were explicitly informed that they had to establish and consolidate the association between the texture of the central cue and the position of the target.

In the training session we used four longer SOAs $(150,250,350$, and $450 \mathrm{~ms}$ ) as compared to pre- and post-training sessions in order to allow more time for the decoding of the cue. SOAs were randomly presented with equal probability in each session. The directional meaning of the textures was counterbalanced across participants. Half of the participants had to associate rhombus-right and square-left, while the reverse association was assigned to the other half.

Each training session consisted of 8 practice trials followed by 2 blocks of 80 trials each. No catch trials were presented in the training sessions. The participants could take a break after each block. The whole training session lasted about 15 minutes including instructions and short breaks. All participants began on a Monday with the pretraining session and with the first training session and carried out the training sessions for 5 consecutive days for 3 weeks with a break for the weekends. On the Friday of the last week they carried out the last training and the post-training session.

In order to check that participants performed in the training sessions as instructed accuracy and RTs during training were evaluated in every subject. Accuracy was calculated as a percentage of correct responses in the target discrimination. Omissions (no responses) were classified as errors and excluded from the analyses. Two independent ANOVAs were carried out on accuracy and RTs, with SOA (150, 250, 350, and $450 \mathrm{~ms}$ ) and Training day (first, second, third, etc. until fifteenth) as within-subject factors.

To evaluate whether training produced an improvement in the cueing effects induced by texture cues a comparison was performed between pre- and post-training sessions. As in Experiment 2, false alarms and omissions were classified as errors and excluded from the analyses. Each type of error accounted for less than 3.07\% of the total 
number of trials. Because these rates were low errors were not analyzed further. Too short or too long RTs (RT $>$ or $<$ at \pm 2 standard deviation cut-off procedure) were also excluded from further analysis. An ANOVA was carried out on mean RT, with Task session (pretraining and post-training), SOA (50,100, 150 or $200 \mathrm{~ms}$ ) and Trial Type (valid and invalid trials) as within-subject variables. In all these analyses, selected two-sample comparisons were performed by means of $t$ tests for paired data, with Bonferroni correction as appropriate, in case of multiple comparisons.

\section{Results}

\subsection{Training}

On the whole, both accuracy and RT improved at the end of the training in comparison with the beginning. In particular, participants reached the top of performance at the seventh day and maintained it until the last day.

Participants showed an accuracy higher than 93\% already at the third day of training which increased to reach a mean accuracy of $96 \%$ in the last nine days. However, a significant difference in accuracy was observed only comparing the first day with most of the days comprised between the fifth and the fifteenth, as revealed by the effect of Training Day $F(14,70)=2.94$, MS $=0.0198, p=0.001$. No other significant main effect or interactions were found.

The ANOVA conducted on RT showed a significant main effect of Training Day $F(14,70)=19.89$, MS $=42790, p<0.00001$, with RTs of the first five days significantly slower than those of most of the subsequent days. No significant improvement in RT was observed in the last nine days. The interaction SOA $\times$ Training Day, $F(42,210)=1.93, \mathrm{MS}=370$, $p=0.001$ was also significant and confirmed that this improvement was present at all the SOAs. The analysis revealed also a significant main effect of SOA, $F(3,15)=92.01$, MS $=69797, p<0.00001$ showing a reduction of $\mathrm{RT}$ at increasing intervals.

\subsection{Pre- versus post-training sessions}

The analysis performed on the pre- and post-training sessions revealed that participants oriented attention according to the learned texture-direction association in the post- but not in the pre-training session. This improvement in the capacity of orienting attention did not apply to all the SOAs but only to the two longest ones $150 \mathrm{~ms}$ $(p=0.01)$ and $200 \mathrm{~ms}(p=0.04)$. At 50 and $100 \mathrm{~ms}$ of SOA no cueing effect was observed neither in the pre- nor in the post-training session (see Fig. 6 and Table 3). ${ }^{1}$ These results were reinforced by the significant effects of Trial Type $F(1,5)=6.69$, MS $=599, p=0.049$, SOA $\times$ Trial Type $F(3,15)=13.52, M S=442, p=0.0001$ and specifically Task Session $\times \mathrm{SOA} \times$ Trial Type $F(3,15)=4.03$, MS $=171, p=0.027$. Also the main effect of SOA was significant indicating that RTs were faster as the SOAs increased. For the Task Session factor no main effect or interaction approached significance.

\section{Discussion}

The results of Experiment 3 provide for the first time evidence that practice improves the ability to shift attention according to the meaning of centrally presented symbolic cues even when they do not have any intrinsic directional significance. There were no cueing effects in the pre-training session while after a training of 15 days a facilitation effect was observed for the predicted locations at 150 and $200 \mathrm{~ms}$ of SOA. Importantly, this improvement does not reflect a mere

\footnotetext{
${ }^{1}$ We examined separately RTs of the three naïve participants and we observed the same trend as that reported with the entire group, with a post-training difference between valid and invalid trials larger than the pre-training difference: $9 \mathrm{~ms}$ at SOA of 150 and $15 \mathrm{~ms}$ at SOA of $200 \mathrm{~ms}$, but not earlier.
}
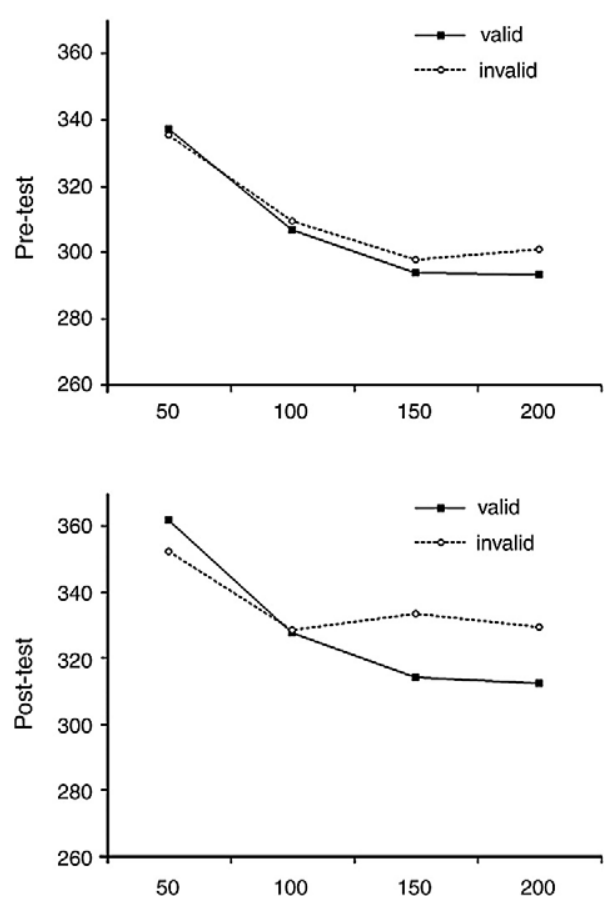

Fig. 6. Mean reaction times (RT) for the texture cues as a function of cue-target stimulus onset asynchrony (SOA) and trial type (valid and invalid trials) for the pre- and posttraining sessions. In valid and invalid trials targets occurred respectively at the location where eyes were looking or arrows were pointing and opposite to them.

reduction in RT since in the post-training session participants were not altogether faster. On the contrary, after training, only a cueing effect, that is, the RT difference between valid and invalid trials was increased.

The training used in this experiment was aimed (a) to familiarize participants with the textures themselves in order to speed up the decoding of the cues, and (b) to induce a conditioning effect between a central texture and a peripheral target with the purpose to associate the central texture to an automatic-like orienting of attention. Both these mechanisms are thought to be responsible for the attentional orienting induced by eyes and arrows. Considering that texture cues, which are novel non-directional cues, were able to induce a very fast orienting of attention after training, the results of this experiment support the importance of learning in the automatic-like attentional effects induced by over-learned central cues. Actually, the cueing effects induced by eyes and arrows in Experiment 2 were earlier than those obtained here. This difference may be explained by the duration of the training in the sense that in this experiment training lasted 3 weeks while with eyes and arrows as natural cues training starts early in life. Further experiments might show that a longer training with symbolic novel cues result in a earlier cueing effect.

Table 3

Mean response times (ms) and standard deviations (SD) for texture cues for each trial type and SOA in the pre- and post-training sessions.

\begin{tabular}{|c|c|c|c|c|}
\hline \multicolumn{5}{|l|}{ SOA } \\
\hline \multirow[b]{2}{*}{ Trial type } & \multirow{2}{*}{$\frac{50}{\text { Mean (SD) }}$} & \multirow{2}{*}{$\frac{100}{\text { Mean (SD) }}$} & \multirow{2}{*}{$\frac{150}{\text { Mean (SD) }}$} & \multirow{2}{*}{$\frac{200}{\text { Mean (SD) }}$} \\
\hline & & & & \\
\hline \multicolumn{5}{|l|}{ Pre-test } \\
\hline Valid & $337.6(26.4)$ & $307.2(24.3)$ & $294.4(24.6)$ & $293.7(23.8)$ \\
\hline Invalid & $335.8(26.8)$ & $309.8(23.2)$ & $297.1(24.6)$ & $301.3(23.4)$ \\
\hline \multicolumn{5}{|l|}{ Post-test } \\
\hline Valid & $362(17.6)$ & $327.9(16)$ & $314.3(15.5)$ & $312.6(15)$ \\
\hline Invalid & $352.4(18.3)$ & $328.9(16)$ & 333.7 (15.5) & 329.8 (17) \\
\hline
\end{tabular}




\section{General discussion}

The aim of the present study was to investigate the cueing effects induced by centrally presented eye and arrow cues. In Experiment 1 , using a counter-predictive paradigm, we evaluated if volitional control can contrast to a similar extent attentional orienting triggered by eyes and arrows. Previous evidence obtained with counterpredictive paradigms has shown that eyes, but not arrows, induce an automatic-like shift of attention. Some methodological aspects, however, may have biased those results. Our procedure, providing a homogeneous perceptual saliency of the two kinds of cues and a direct comparison of their effects, enabled to conclude that volitional control exerts a similar effect on the attentional shift induced by the two cues with the same time course. When participants were instructed to orient their attention to the location opposite to that indicated by eyes and arrows they were able to do it very quickly starting as early as $100 \mathrm{~ms}$ of SOA. The control system in the presence of counterpredictive eyes and arrows was rapidly set in order to avoid the irrelevant information so as to improve its performance. Interestingly, this early ability to voluntarily orient attention towards a spatial location cannot be the result of practice during the experimental session but is to be ascribed to an early action of voluntary control. Other studies suggested that the control of attention may be more profoundly influenced by volitional factors than it was classically thought and that this may occur early in time even in the presence of peripheral cues (Folk et al., 1992; Warner et al., 1990). The results of Experiment 1, thus, suggest that despite their biological meaning the eyes are not unique in triggering attentional shifts. This does not mean to deny that gaze is a special stimulus. Gaze perception is an innate condition as shown by the fact that 2-3 months old infants can attend to eyes and faces (Maurer, 1985) and begin to follow the gaze direction within their first year of life (e.g. D'Entremont, Hains, \& Muir, 1997). Moreover, neuropsychological and neuroimaging evidence in humans (Allison, Puce, \& McCarthy, 2000; Allison, Puce, Spencer, \& McCarthy, 1999; Bentin, Allison, Puce, Perez, \& McCarthy, 1996; Damasio, Damasio, \& Van Hoesen, 1982; Hoffman \& Haxby, 2000; Klin, Jones, Schultz, Volkmar, \& Cohen, 2002; Puce, Allison, Bentin, Gore, \& McCarthy, 1998; Puce \& Perrett, 2003; Wicker, Michel, Henaff, \& Decety, 1998), as well as single-cell studies in monkeys (Brothers, 1995; Bruce, Desimone, \& Gross, 1981; Perrett \& Emery, 1994; Perrett, Harries, Mistlin, Hietanen, Benson, \& Bevan, 1990; Perrett, Rolls, \& Caan, 1982), highlight the presence of a specific temporal lobe area (i.e., the superior temporal sulcus) that is involved in gaze processing. However, even if different neuronal mechanisms are presumably involved in the visual processing of gaze and arrows it is entirely possible that the subsequent orienting of attention is based on the same neuronal network. Recent event-related potential (ERP) data from our study (Brignani et al., 2009) provided evidence consistent with the hypothesis that eyes and arrows share the same cortical network during a spatial cueing paradigm. One should notice, however, that there is no agreement about the neurophysiological mechanisms which subtend these cueing effects. Hietanen et al. (2006), for instance, conjointly using functional magnetic resonance imaging (fMRI) and ERPs, concluded that the attentional orienting induced by eye and arrow cues is subserved by two partially overlapping, but different, cortical networks. Further investigations are needed to clarify this point.

From a behavioural point of view, eyes and arrows are both able to induce a very fast orienting of attention when centrally presented. In Experiment 2 we compared these two cues with a novel cue requiring an arbitrary texture-direction association and thus an entirely voluntary control. The results showed that eye and arrow cues were able to orient attention much earlier (i.e., starting from $50 \mathrm{~ms}$ of SOA) in comparison with the novel cue. Eyes and arrows, as well as faces, manual gestures and direction words, are communicative signals widely used in daily life. It has been recently proposed that all conventional communicative signals induce an automatic-like orient- ing of attention as a consequence of an over-learned association mechanism between the symbols and the locations to which they refer (Hommel et al., 2001; Vecera \& Rizzo, 2004, 2006). Consequently, the directional meaning of communicative signals is thought to be processed very quickly and to directly affect the spatial orienting of visual attention. It is interesting to note that children can interpret and use the directional meaning of arrow cues even when they are very young (three years old) (Ristic et al. (2002). Hommel et al. (2001) reported that both non-predictive arrows and direction words (i.e., right, left) may induce a facilitation in the processing of a target. This supports the notion that over-learned symbols can direct the visual attention of human observers in a relatively automatic fashion even when such symbols are not biologically relevant. On the basis of evidence from a patient with frontal lobe damage which was able to orient with peripheral but not with central symbolic cues Vecera and Rizzo (2004, 2006) suggested that the orbitofrontal cortex may be the biological basis of this over-learned association mechanism. Consistently with this hypothesis, in our study (Brignani et al., 2009) on the electrophysiological correlates of the attentional shift induced by arrows and eyes we found the involvement of a cortical network which likely included the orbitofrontal cortex.

In order to test the importance of learning in the attentional orienting induced by these communicative signals we carried out Experiment 3 in which participants were trained for three weeks to consolidate a new symbol-direction association. If practice holds a role in this mechanism we expected to observe an improvement in the ability to shift attention after training. Indeed, while participants were not able to shift attention according to the directional meaning of the texture before the training session, they were able to do so after training. Thus, practice was successful in improving the time course of voluntary orienting of attention with a novel arbitrary cue. These data support the importance of learning mechanisms in the attentional shifts induced by over-learned cues, such as eyes and arrows.

However, the cueing effects induced by this novel cue were not as early as those observed with eyes and arrows in the previous two experiments. This difference may be ascribed to the duration of the training period. A longer practice, indeed, could have produced earlier attentional shifts, but another factor, which concerns the perceptual features of the cues, could also account for this discrepancy. The texture cues, for instance, are more complex stimuli and thus could be more difficult to distinguish from each other than leftward or rightward eyes and arrows. These latter, in addition, show an intrinsic spatial correspondence between some of their features and the target location which they indicate. In both, for instance, there are high-luminance (white) regions which are located ipsilaterally in arrows and contralaterally in eyes relative to the cued direction. It may be supposed that participants used these features for the attentional shift before processing the cue as a whole. This hypothesis may also explain the early advantage ( $100 \mathrm{~ms}$ ) for the predictive, although spatially incongruent, positions observed in Experiment 1 with counter-predictive cues. To eliminate this possibility the novel texture cues used in Experiment 3 were perfectly symmetric and showed no correspondence between any feature and the cued location of the target. It may be that very early cueing effects (i.e., 50 and $100 \mathrm{~ms}$ of SOA) are evoked only when the cue is asymmetric allowing spatial correspondence between central cue and target location to be automatically paired. Recent studies showed that spatial correspondence may help to develop fast orienting effects (Lambert \& Duddy, 2002; Lambert, Roser, Wells, \& Heffer, 2006) but further investigations are necessary to evaluate the link between learning mechanisms and cues asymmetry and their role in the attentional effects induced by eyes and arrow cues.

In summary, the results of the present study show that both eye and arrow cues induce identical behavioural effects in spatial cueing tasks also when a counter-predictive paradigm is used. Evidence are provided that an over-learned association mechanism plays a relevant role in the establishment of these attentional effects. 


\section{References}

Allison, T., Puce, A., \& McCarthy, G. (2000). Social perception from visual cues: Role of the STS region. Trends Cogn Sci, 4(7), 267-278.

Allison, T., Puce, A., Spencer, D. D., \& McCarthy, G. (1999). Electrophysiological studies of human face perception. I: Potentials generated in occipitotemporal cortex by face and non-face stimuli. Cerebral Cortex, 9(5), 415-430.

Bentin, S., Allison, T., Puce, A., Perez, E., \& McCarthy, G. (1996). Electrophysiological studies of face perception in humans. J Cogn Neurosci, 8(6), 551-565.

Brignani, D., Guzzon, D., Marzi, C. A., \& Miniussi, C. (2009). Attentional orienting induced by arrows and eye-gaze compared with an endogenous cue. Neuropsychologia, 47(2), 370-381.

Brothers, L. (1995). The neurophysiology of the perception of intentions by primates. In M. S. Gazzaniga (Ed.), The cognitive neurosciences. Cambridge, MA: MIT Press.

Bruce, C., Desimone, R., \& Gross, C. G. (1981). Visual properties of neurons in a polysensory area in superior temporal sulcus of the macaque. J Neurophysiol, 46(2), 369-384.

D'Entremont, B., Hains, S., \& Muir, D. (1997). A demonstration of gaze following in 3- to 6-month-olds. Infant Behavior and Development, 20, 569-572.

Damasio, A. R., Damasio, H., \& Van Hoesen, G. W. (1982). Prosopagnosia: Anatomic basis and behavioral mechanisms. Neurology, 32(4), 331-341.

Driver, J., Davis, G., Ricciardelli, P., Kidd, P., Maxwell, E., \& Baron-Cohen, S. (1999). Gaze perception triggers visuospatial orienting by adults in a reflexive manner. Visual Cognition, 6, 509-540.

Folk, C. L., Remington, R. W., \& Johnston, J. C. (1992). Involuntary covert orienting is contingent on attentional control settings. J Exp Psychol Hum Percept Perform, 18(4), $1030-1044$

Friesen, C. K., \& Kingstone, A. (1998). The eyes have it! Reflexive orienting is triggered by nonpredictive gaze. Psychonomic Bulletin E' Review, 5(3), 490-495.

Friesen, C. K., \& Kingstone, A. (2003). Abrupt onsets and gaze direction cues trigger independent reflexive attentional effects. Cognition, 87(1), B1-B10.

Friesen, C. K., Ristic, J., \& Kingstone, A. (2004). Attentional effects of counterpredictive gaze and arrow cues. J Exp Psychol Hum Percept Perform, 30(2), 319-329.

Frischen, A., \& Tipper, S. P. (2004). Orienting attention via observed gaze shift evokes longer term inhibitory effects: Implications for social interactions, attention, and memory. J Exp Psychol Gen, 133(4), 516-533.

Frischen, A., Smilek, D., Eastwood, J. D., \& Tipper, S. P. (2007). Inhibition of return in response to gaze cues: The role of time-course and fixation cue. Visual Cognition, 15 $881-895$.

Gibson, B. S., \& Bryant, T. A. (2005). Variation in cue duration reveals top-down modulation of involuntary orienting to uninformative symbolic cues. Percept Psychophys, 67(5), 749-758.

Hietanen, J. K., \& Leppanen, J. M. (2003). Does facial expression affect attention orienting by gaze direction cues? J Exp Psychol Hum Percept Perform, 29(6), $1228-1243$.

Hietanen, J. K., Nummenmaa, L., Nyman, M. J., Parkkola, R., \& Hamalainen, H. (2006). Automatic attention orienting by social and symbolic cues activates different neural networks: An fMRI study. Neuroimage, 33(1), 406-413.

Hoffman, E. A., \& Haxby, J. V. (2000). Distinct representations of eye gaze and identity in the distributed human neural system for face perception. Nature Neuroscience, 3(1), $80-84$.

Hommel, B., Pratt, J., Colzato, L., \& Godijn, R. (2001). Symbolic control of visual attention. Psychol Sci, 12(5), 360-365.

Jonides, J. (1981). Voluntary vs. Automatic control over the mind's eye's movement. In J. B. Long, \& A. D. Baddeley (Eds.), Attention and Performance IX. Hillsdale NJ: Lawrence Erlbaum Associates.

Jonides, J., \& Yantis, S. (1988). Uniqueness of abrupt visual onset in capturing attention. Percept Psychophys, 43(4), 346-354.

Klein, R. M. (2000). Inhibition of return. Trends Cogn Sci, 4(4), 138-147.

Klein, R. M., Kingstone, A., \& Pontefract, A. (1992). Orienting of visual attention. In K. Rayner (Ed.), Eye movements and visual cognition:Scene perception and reading (pp. 46-65). New York: Springer-Verlag.

Klin, A., Jones, W., Schultz, R., Volkmar, F., \& Cohen, D. (2002). Visual fixation patterns during viewing of naturalistic social situations as predictors of social competence in individuals with autism. Archives of General Psychiatry, 59(9), 809-816.
Lambert, A., \& Duddy, M. (2002). Visual orienting with central and peripheral precues: Deconfounding the contributions of cue eccentricity, cue discrimination and spatial correspondence. Visual Cognition, 9(3), 303-336.

Lambert, A., Roser, M., Wells, I., \& Heffer, C. (2006). The spatial correspondence hypothesis and orienting in response to central and peripheral spatial cues. Visual Cognition, 13(1), 65-88.

Langton, S. R., \& Bruce, V. (1999). Reflexive social orienting. Visual Cognition, 6, $541-567$.

Langton, S. R., Honeyman, H., \& Tessler, E. (2004). The influence of head contour and nose angle on the perception of eye-gaze direction. Percept Psychophys, 66(5), $752-771$.

Maurer, D. (1985). Infants' perception of facedness. In T. Field \& N. Fox (Eds.), Social perception in infants (pp. 73-100). Norwood, NJ: Ablex.

Muller, H. J., \& Findlay, J. M. (1988). The effect of visual attention on peripheral discrimination thresholds in single and multiple element displays. Acta Psychol (Amst), 69(2), 129-155.

Muller, H. J., \& Rabbitt, P. M. (1989). Reflexive and voluntary orienting of visua attention: time course of activation and resistance to interruption. J Exp Psychol Hum Percept Perform, 15(2), 315-330.

Oldfield, R. C. (1971). The assessment and analysis of handedness: The Edinburgh inventory. Neuropsychologia, 9(1), 97-113.

Perrett, D., \& Emery, N. (1994). Understanding the intentions of others from visual signals: Neurophysiological evidence. Cahiers de Psychologie Cognitive, 13, 683-694.

Perrett, D. I., Harries, M. H., Mistlin, A. J., Hietanen, J. K., Benson, P. J., Bevan, R., et al. (1990). Social signals analyzed at the single cell level: Someone is looking at me, something touched me, something moved! International Journal of Comparative Psychology, 4, 25-55.

Perrett, D. I., Rolls, E. T. \& Caan, W. (1982). Visual neurones responsive to faces in the monkey temporal cortex. Experimental Brain Research, 47(3), 329-342.

Posner, M. I., Snyder, C. R., \& Davison, B. J. (1980). Attention and the detection of signals J Exp Psychol, 109(2), 160-174.

Posner, M. I. (1980). Orienting of attention. Q

Posner, M. I., Cohen, Y., \& Rafal, R. D. (1982). Neural systems control of spatial orienting. Philosophical Transactions of the Royal Society of London. Series B: Biological Sciences, 298(1089), 187-198.

Puce, A., Allison, T., Bentin, S., Gore, J. C., \& McCarthy, G. (1998). Temporal cortex activation in humans viewing eye and mouth movements. J Neurosci, 18(6) $2188-2199$.

Puce, A., \& Perrett, D. (2003). Electrophysiology and brain imaging of biological motion. Philosophical Transactions of the Royal Society of London. Series B: Biological Sciences, 358(1431), 435-445.

Ristic, J., Friesen, C. K., \& Kingstone, A. (2002). Are eyes special? It depends on how you look at it. Psychon Bull Rev, 9(3), 507-513.

Teichner, W. H. (1954). Recent studies of simple reaction time. Psychological Bulletin, 51 $(2: 1), 128-149$

Tipples, J. (2002). Eye gaze is not unique: Automatic orienting in response to uninformative arrows. Psychon Bull Rev, 9(2), 314-318.

Tipples, J. (2008). Orienting to a counterpredictive gaze and arrow cues. Percept Psychophys, 70(1), 77-87.

Vecera, S. P., \& Rizzo, M. (2004). What are you looking at? Impaired 'social attention' following frontal-lobe damage. Neuropsychologia, 42(12), 1657-1665.

Vecera, S. P., \& Rizzo, M. (2006). Eye gaze does not produce reflexive shift of attention evidence from frontal-lobe damage. Neuropsychologia, 44(1), 150-159.

Warner, C. B., Juola, J. F., \& Koshino, H. (1990). Voluntary allocation versus automatic capture of visual attention. Percept Psychophys, 48(3), 243-251.

Wicker, B., Michel, F., Henaff, M. A., \& Decety, J. (1998). Brain regions involved in the perception of gaze: A PET study. Neuroimage, 8(2), 221-227.

Yantis, S., \& Jonides, J. (1984). Abrupt visual onsets and selective attention: Evidence from visual search. J Exp Psychol Hum Percept Perform, 10(5), 601-621.

Yantis, S., \& Jonides, J. (1990). Abrupt visual onsets and selective attention: Voluntary versus automatic allocation. J Exp Psychol Hum Percept Perform, 16(1), 121-134. 